\title{
Relevance of Physical Activities, Sedentary Behaviors, and Genetic Predisposition in Body Fatness: Population-Based Study on Chinese Adults
}

\author{
Hongmei Xue ${ }^{a}$ b Xiao Zhang ${ }^{b}$ Danting Li ${ }^{b}$ Mengxue Chen ${ }^{b}$ Jiao Luo ${ }^{b}$ \\ Yunhui Gong ${ }^{c}$ Xiaohua Lv ${ }^{b}$ Liming Quan $^{b}$ Fang He $^{b}$ Lishi Zhang ${ }^{b}$ \\ Guo Cheng ${ }^{\text {b }}$ \\ aCollege of Public Health, Hebei University, Key Laboratory of Public Health Safety of Hebei University, Baoding, \\ China; b' West China School of Public Health, Sichuan University, Chengdu, China; 'West China Second University \\ Hospital, Sichuan University, Chengdu, China
}

\section{Keywords}

Sedentary time - Physical activities - Genetic predisposition . Body fat

\begin{abstract}
Introduction: Little attention has been paid to the interacting effect of specific intensities of physical activities (PAs) and sedentary lifestyle, like television watching, and genetic predisposition on body composition indices among Chinese adults. Herein, we aimed to examine whether specific types of PAs and sedentary behaviors (SBs) were associated with body composition indices among Chinese adults and to further explore whether these associations interacted with the genetic predisposition to high BMI. Methods: Cross-sectional data regarding PAs and time spent on SBs and dietary intake of 3,976 Chinese adults (54.9\% women) aged 25-65 years in Southwest China were obtained via questionnaires in 2013-2015. Weight, height, and waist circumference (WC) were measured, and $\mathrm{BMI}$, percentage of body fat (\%BF), fat mass index (FMI), and fat-free mass index (FFMI) of the participants were calculated. Genetic risk score (GRS) was calculated on 9 established BMI-associated SNPs among Chinese
\end{abstract}

karger@karger.com www.karger.com/ofa

Karger $\stackrel{\text { ' }}{5}$

BOPEN ACCESS
(C) 2021 The Author(s)

Published by S. Karger AG, Basel

This is an Open Access article licensed under the Creative Commons Attribution-NonCommercial-4.0 International License (CC BY-NC) (http://www.karger.com/Services/OpenAccessLicense), applicable to the online version of the article only. Usage and distribution for commercial purposes requires written permission. adults. Results: When the participants were stratified by GRS for BMI, significant associations were only found for adults with high GRS for BMI: moderate-to-vigorous physical activity (MVPA) was negatively associated with WC and \%BF and positively related to FFMI. The adjusted positive relationship of time spent watching television with $\mathrm{BMI}, \mathrm{WC}, \% \mathrm{BF}$, and FMI were also just found between adults with high weighted GRS for high BMl: for every $1 \mathrm{~h}$ increment in television watching, the $\mathrm{BMI}, \mathrm{WC} \% \mathrm{BF}$, and $\mathrm{FMl}$ of the participants increased by $0.2 \mathrm{~kg} / \mathrm{m}^{2}, 0.9 \mathrm{~cm}, 0.3 \%$, and $0.1 \mathrm{~kg} / \mathrm{m}^{2}$, respectively $(p<$ 0.02 ). Conclusion: MVPA may be a protective factor against obesity, and prolonged television watching may accentuate adiposity. These putative effects may be more pronounced among individuals with a high genetic risk of a high BMI.

(c) 2021 The Author(s).

Published by S. Karger AG, Basel

\section{Introduction}

Obesity and related comorbid conditions, such as cardiovascular disease, hypertension, type 2 diabetes, and cancer, as well as mortality, have become a serious threat
Correspondence to:

Hongmei Xue, xhmei_1109@163.com 
to public health globally [1]. Obesity is a multifactorial disease, both genetic predisposition and environmental factors are important contributors to adulthood obesity $[1,2]$. The well-accepted viewpoint is that genetic factors cannot explain its pathogenesis independently, while genes-environment interaction can ultimately induce the disease. Physical activities (PAs) and sedentary behaviors (SBs) are important and modifiable environmental factors, and it is of great theoretical and practical significance to study the association between PAs or SBs and adulthood obesity with consideration of genetic predisposition.

Epidemiological studies from China, the USA, and Denmark, and so on, have shown that lack of PAs and excess SBs were associated with an increased risk of obesity [3]. Research studies in Western countries have suggested that a sedentary lifestyle, indicated by prolonged television watching, may be a risk factor for adulthood obesity independent of the practice of PAs [4-7]. However, few studies focused on the interactions between PAs or SBs and genetic predisposition to adulthood obesity. Existing studies in Western countries have shown that engaging in PAs may reduce the upward tendency toward obesity [3]. Additionally, 2 studies on Chinese adults [8, 9] have suggested that adopting a physically active lifestyle may help to reduce the genetic influence on BMI among the Chinese population. However, less attention has been paid to the effects of PA intensity, which may have a different effect on obesity [10].

Thus far, only 2 studies -1 from the USA [11] and 1 from Finland [12] - have investigated the interacting effect of television watching and genetic predisposition on body composition indices. The US study [11] showed that prolonged television viewing by women aged $30-55$ years and men aged 40-75 years may increase the tendency to have a higher BMI. The study focused on Finnish adults aged 30-45 years [12] showed a positive correlation between television viewing and BMI and waist circumference (WC), even after adjusting for the genetic risk score (GRS) for obesity [7].

In China, nearly $83 \%$ of Chinese adults spend 2 or more hours a day engaged in sedentary activities, and $82 \%$ do not meet the recommendation of the World Health Organization (WHO) of $2.5 \mathrm{~h}$ per week of moderate-intensity PAs [13]. Additionally, 9 SNPs were found to be significantly associated with obesity in the Chinese population on a genome-wide scale in 2 meta-analyses $[14,15]$. Limited research was observed on the interacting effect of PAs and genetic predisposition on adulthood obesity, and we are not aware of any studies examining the interacting effect of SBs and genetic predisposition on obesity among Chinese adults. Given the limited research, adults from an ongoing, population-based, prospective study in Southwest China were identified to examine whether specific types of PAs and SBs were associated with body composition indices among Chinese adults, and to further explore whether these associations interacted with a genetic susceptibility to having a high BMI.

\section{Materials and Methods}

\section{Study Sample}

We used data from the baseline survey of the Nutrition and Health in Southwest China (NHSC) (adults aged 18-70 years) beginning in September 2013, which aims to investigate the health impact of nutritional and lifestyle factors on the development of several chronic diseases or worsening of life quality, as described elsewhere [16]. Briefly, 29 study sites (10 communities and 19 villages) were included until 2015. At each site, a 2-stage (householdperson) sampling was used. Adults who agreed to participate in the cohort study were invited to the study center for interviews. Generally, each visit included anthropometric measurements, medical examinations, questionnaires, and face-to-face interviews by trained investigators about nutrition-related behaviors, lifestyles, and social status. All participants were followed biennially to obtain updated information. Participants who were cooperative, who volunteered, and who signed an informed consent form were included in this cohort study. The study protocol was approved by the Ethics Committee of West China School of Public Health (No. 4 West China Teaching Hospital) of Sichuan University (approved date: March 3, 2014). All participants provided written informed consent for all examinations.

\section{Patient and Public Involvement}

Eligible data in the present analysis were identified from the baseline survey between 2013 and 2015. Participants in the present analysis have been shown to be comparable to age-matched adults in our cohort study and the general population of urban and rural areas in Southwest China in terms of sociodemographic and lifestyle characteristics [17]. Initially, 4,708 adults aged 25-65 years were identified. Of these, participants with incomplete information (missing data regarding anthropometry indices or other important confounding variables $[n=145]$ ); an implausible energy intake (EI) [18] given on food frequency questionnaires (FFQs) $(n=237)$; and diabetes, cardiovascular diseases, or cancer $(n=350)$ were excluded. Therefore, a sample of 3,976 Chinese adults was used in the present analysis to examine the associations between specific types of PAs, SBs, and body composition, and to explore whether the relationship between specific types of PAs and SBs and body composition indices was interacted with GRS for a high BMI.

\section{SBs and PAs}

Assessment of SBs and PAs was described previously [19]. In brief, participants were asked about typical type, yearly/monthly/ weekly/daily frequency, and duration of moderate- to vigorousintensity PAs and SBs (television watching and using computer/ phone while sitting, etc.). Based on the above data, the mean daily time spent on each type of SB was estimated in hours by multiply- 
ing frequency and duration and dividing by 1 year/month/week/ day. Total SB time was calculated as a sum of time spent on sedentary activities, and screen-based SB time as a sum of time spent on television watching and computer/phone using. To quantify the intensity of PAs, metabolic equivalent tasks (METs) were used according to a 2011 update of a published compendium, organized by MET category [20]. Based on the above information, daily energy expenditure on moderate-to-vigorous physical activity (MVPA: METs $\geq 3$ ), moderate physical activity (MPA: METs $\geq 3$ and METs $\leq 6$ ), and vigorous physical activity (VPA: METs $\geq 6$ ) $[20,21]$ was calculated by multiplying the body weight in kilograms by the METs value and duration of activities per day.

\section{Anthropometric Measurements}

Anthropometric measurements were carried out by trained medical personnel in each research center following standard procedures. Weight and height were measured using an ultrasonic instrument (Weight and Height Instrument DHM-30; Dingheng Ltd., Zhengzhou, China), which was accurate to the nearest $0.1 \mathrm{~kg}$ and $0.1 \mathrm{~cm}$, respectively. WC was measured on standing participants at the midway point between the lowest rib and the iliac crest to the nearest $0.1 \mathrm{~cm}$ using a nonelastic tape measure. Weight, height, and WC were each averaged based on 2 measurements.

For this analysis, BMI was calculated by dividing the weight in kilograms by the height in meters squared. Being overweight was defined according to WHO standards (BMI $\geq 25 \mathrm{~kg} / \mathrm{m}^{2}$ ) [22]. The percentage of body fat $(\% \mathrm{BF})$ was calculated using the equations of Liu et al. [23]. Moreover, the fat mass index (FMI) and the fatfree mass index (FFMI) were calculated using the following equations [24]: FMI $\left(\mathrm{kg} / \mathrm{m}^{2}\right)=($ weight $\times \% \mathrm{BF}) /$ height $^{2}$ and FFMI $(\mathrm{kg} /$ $\left.\mathrm{m}^{2}\right)=($ weight $\times[1 / \% \mathrm{BF}]) /$ height $^{2}$.

\section{Genotyping and GRS Computation}

A peripheral blood specimen was collected from each participant following an overnight (from midnight) fast. Genomic DNA was extracted from peripheral blood lymphocytes through the standard procedure (DP-318, Tiangen Biotech (Beijing) Co., Ltd., China). The DNA samples were genotyped using Infinium II technology from Illumina (Human HAP300 panel). Furthermore, the genotype frequency distributions were consistent with the HardyWeinberg equilibrium $(p>0.01)$ [25].

We selected 9 SNPs that showed significant genome-wide associations with BMI among Chinese Han populations [14, 15]. The GRS was calculated based on the 9 established BMI-associated genetic variants using a previously reported weighted method

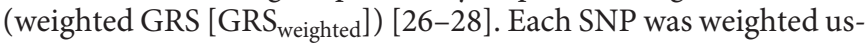
ing its relative effect size ( $\beta$ coefficient), which was obtained from the reported meta-analysis data in Chinese adults $[14,15]$.

\section{Assessment of Covariates}

Participants were asked to complete a self-administered questionnaire collecting information about their family characteristics and educational and employment status. For the present analysis, we assessed factors that were potentially associated with television watching and body composition, which included gender, age (years), smoking status (current smoking, no longer smokes, and never smoked), alcohol intake (yes or no), education level $(<6$ years, 6-12 years, and $>12$ years of schooling), occupation (yes or no), monthly family income $(<1,000$ Chinese yuan [CNY], $\geq 1,000$ $3,000 \mathrm{CNY}, \geq 3,000-6,000 \mathrm{CNY}$, and $\geq 6,000 \mathrm{CNY}$ ), and monthly personal income $(<1,000$ Chinese yuan [CNY], $\geq 1,000-3,000$ $\mathrm{CNY}, \geq 3,000-6,000 \mathrm{CNY}$, and $\geq 6,000 \mathrm{CNY}$ ).

The detailed dietary information was assessed through FFQs in face-to-face interviews. Dietary intake data from the FFQs were converted into energy and nutrient data using the continuously updated in-house nutrient database based on NCCW software (version 11.0, 2014; Qingdao University Medical College, Shandong, China), which reflected the China Food Composition [29]. In this analysis, the total EI for each participant was calculated in kilocalories per day.

Statistical Analyses

SAS procedures (SAS, version 9.3, 2011; SAS Institute Inc., Cary, NC, USA) were used for the data analyses. All analyses were performed using significance level at $p<0.05$, except for interaction tests, in which $p<0.1$ was considered significant. Based on preliminary analyses, which indicated differentiated obesity GRSweighted associations between the PAs, SBs, and body composition $(p<0.1)$, we analyzed the data in terms of the low-, moderate-, and high-obesity GRS $_{\text {weighted }}$ separately.

The time spent watching television was grouped into tertiles (T1-T3) to illustrate its association with the general characteristics. Significant differences for normally distributed continuous variables were analyzed using ANOVAs, while non-normally distributed continuous variables were analyzed via the Kruskal-Wallis tests, and the $\chi^{2}$ tests were used for categorical variables.

Multivariable linear generalized regression models were used to investigate the independent associations between television watching and the body composition indices. Each PA intensity (MVPA, MPA, and VPA) and the specific types of SB (time spent watching television, using the computer/phone, screen-based SB, and total sedentary time) were defined as the independent variables. Moreover, BMI, WC, \%BF, FMI, and FFMI, as measures of body composition, were the dependent variables in separate models. Because of the skewed distributions of BMI, \%BF, FMI, and FFMI, the natural logarithm of these variables was calculated to improve the normality. In the basic models, the analysis of the correlations between each intensity of PA (MVPA, MPA, and VPA), specific types of SB (time spent watching television, using the computer/phone, screenbased SB, and total sedentary time), and the body composition indices was carried out first. In a further step, the following potential covariates affecting these associations were added: gender, age, education level, occupation, average family monthly income, monthly personal income, sleep duration, smoking status, EI, and MVPA energy expenditure as part of the analysis of each $\mathrm{SB}$, as well as the total SBs in the analysis of each MVPA energy expenditure. Each variable was initially considered separately; only variables that had an independent significant effect on the basic models or that substantially modified the principal associations of time spent on SBs and MVPA energy expenditure with body composition measures were included in the subsequent multivariable analyses.

\section{Results}

The general characteristics of the sample in this study, stratified by tertiles of GRS weighted for BMI, are presented in Table 1. Of the adults in the present analysis, 
Table 1. Characteristics ${ }^{1}$ of the study sample by $\operatorname{GRS}_{\text {weighted }}$ for BMI $(n=3,976)$

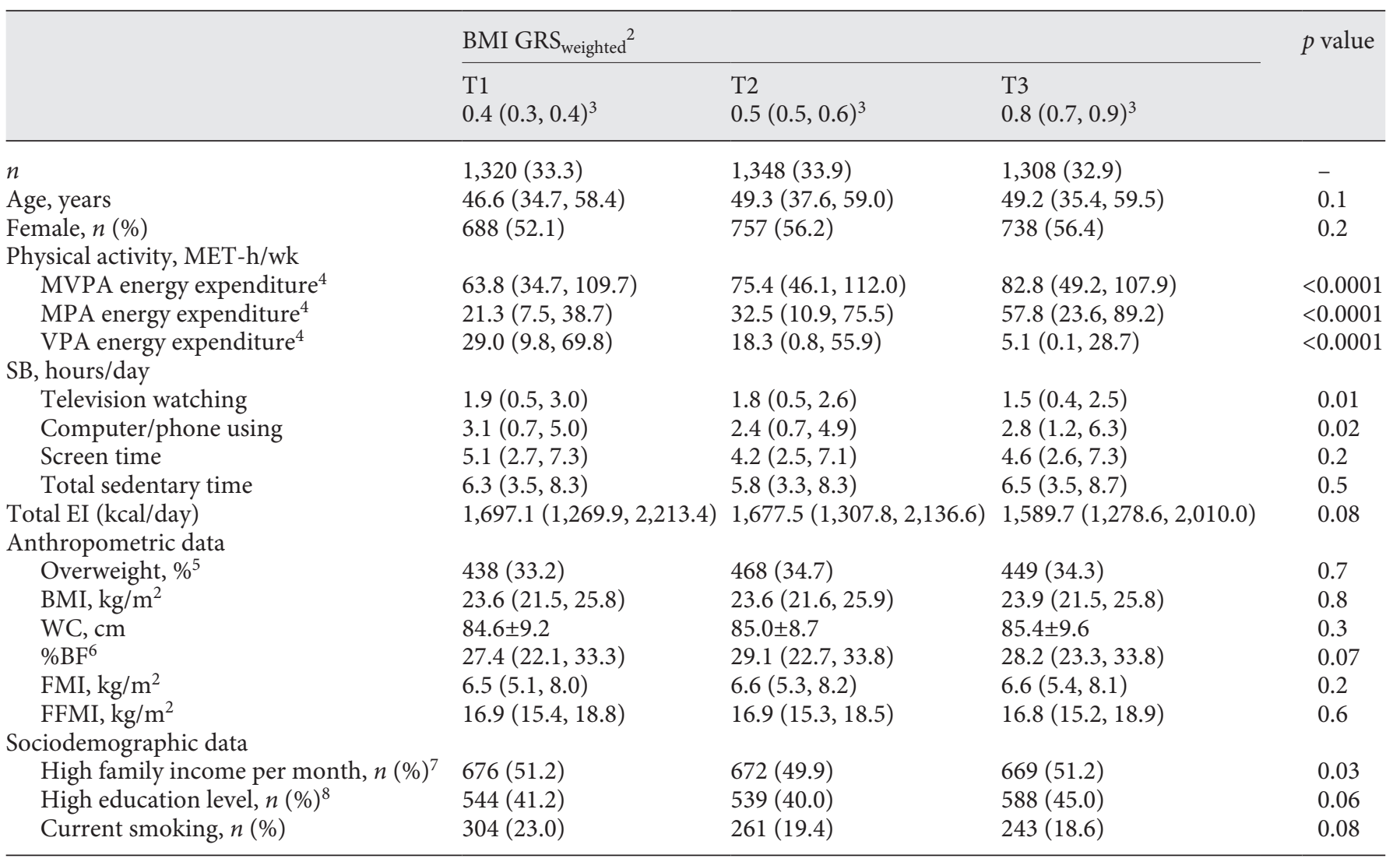

$\mathrm{GRS}_{\text {weighted }}$, weighted genetic risk score; SB, sedentary behavior; EI, energy intake; WC, waist circumference; FMI, fat mass index; FFMI, fat-free mass index; \%BF, percentage of body fat; MVPA, moderate-to-vigorous physical activity; MPA, moderate physical activity; MET, metabolic equivalent task; VPA, vigorous physical activity. ${ }^{1}$ Values are given as median (25th percentage and 75 th percentage) for non-normally distributed continuous variables, mean \pm SD for normally distributed continuous variables, or frequencies for categorical variables. The significant differences between tertiles were tested using the Kruskal-Wallis tests for non-normally distributed continuous variables, ANOVA for normally distributed continuous variables, and the $\chi^{2}$ tests for categorical variables. ${ }^{2}$ Ranges for tertiles (T) 1 through 3; GRS weighted for BMI. ${ }^{3}$ Values are given as median (25th percentage and 75th percentage). ${ }^{4} \mathrm{MVPA}$ energy expenditure: energy expended on MVPAs (MET-h/wk); MPA energy expenditure: energy expended on MPAs (MET-h/wk); VPA energy expenditure: energy expended on VPAs (MET-h/wk) $[15,16] .{ }^{5} \mathrm{BMI} \geq 25 \mathrm{~kg} / \mathrm{m}^{2}$ [17]. ${ }^{6}$ Calculated according to the equations from Liu et al. [18]. ${ }^{7}$ Monthly family income at least $\geq 3,000 \mathrm{CNY}$, which is a moderate level among the general population in Southwest China [12]. ${ }^{8}$ At least 12 years of school education.

$54.9 \%$ were women, and the median age was 48.4 years. Participants with a higher GRS for having a high BMI had higher family income, spent less time watching television and using computer/phone, expended more energy doing MVPA and MPA, and expended less energy doing VPA $(p<0.04)$. When television watching was stratified (Table 2), the participants with a daily television watching time in the highest tertile were older, had a lower personal income and education level, and had a higher BMI, $\mathrm{WC}, \% \mathrm{BF}$, and FMI $(p<0.002)$.

Physical Activities, Sedentary Behaviors, and Body Fat
For the general population in the present study, MVPA was inversely associated with WC after adjusting for gender, age at the baseline, the family's average monthly income, smoking, EI, and total sedentary time. No statistically significant association was observed between MVPA and BMI, \%BF, FMI, or FFMI. Additionally, MPA was negatively associated with $\mathrm{WC}, \% \mathrm{BF}$, and FMI, even after adjusting for all covariates. VPA was negatively related to each of the body composition indices (Table 3). After considering the GRS for a high BMI, positive associations of MVPA, MPA, and VPA with the body composition indices were

Obes Facts 2021;14:346-356 349 
Table 2. Characteristics ${ }^{1}$ of the study sample by television watching time tertiles $(n=3,976)$

\begin{tabular}{|c|c|c|c|c|}
\hline & \multicolumn{3}{|c|}{ Daily time spent on television watching ${ }^{2}$} & \multirow[t]{2}{*}{$p$ value } \\
\hline & $0.0(0.0,0.5)^{3}$ & $1.5(1.0,2.0)^{3}$ & $3.0(2.5,4.0)^{3}$ & \\
\hline$n(\%)$ & $1,282(32.2)$ & $1,372(34.5)$ & $1,322(33.3)$ & - \\
\hline Age, years & $39.9(28.0,52.6)$ & $50.0(39.3,60.1)$ & $52.1(42.0,60.1)$ & $<0.0001$ \\
\hline Female, $n(\%)$ & $647(50.5)$ & $749(54.6)$ & $787(59.5)$ & 0.003 \\
\hline Total EI, kcal/day & $1,663.7(1,308.6,2,078.8)$ & $1,655.29(1,269.3,2,159.9)$ & $1,631.9(1,295.3,2,135.4)$ & 1.0 \\
\hline \multicolumn{5}{|l|}{ Anthropometric data } \\
\hline Overweight, $\%^{6}$ & $383(29.9)$ & $486(35.4)$ & $485(36.7)$ & 0.057 \\
\hline $\mathrm{BMI}, \mathrm{kg} / \mathrm{m}^{2}$ & $23.3(21.0,25.5)$ & $23.8(21.8,25.9)$ & $23.9(21.7,26.0)$ & 0.002 \\
\hline $\mathrm{WC}, \mathrm{cm}$ & $83.6 \pm 9.2$ & $85.6 \pm 9.1$ & $85.7 \pm 9.1$ & $<0.0001$ \\
\hline$\% \mathrm{BF}, \%^{7}$ & $26.6(21.7,32.2)$ & $28.6(22.9,33.8)$ & $30.4(23.4,34.2)$ & $<0.0001$ \\
\hline Occupation: yes, $n(\%)$ & $1,003(78.2)$ & $869(63.4)$ & $773(58.5)$ & $<0.0001$ \\
\hline High education level, $n(\%)^{9}$ & $707(55.1)$ & $528(38.5)$ & $436(33.0)$ & $<0.0001$ \\
\hline Current smoking, $n(\%)$ & $239(18.7)$ & $279(20.3)$ & $289(21.9)$ & 0.7 \\
\hline
\end{tabular}

WC, waist circumference; FMI, fat mass index; FFMI, fat-free mass index; EI, energy intake; \%BF, percentage of body fat; $\mathrm{GRS}_{\text {weighted }}$ weighted genetic risk score; MVPA, moderate-to-vigorous physical activity. ${ }^{1}$ Values are given as median $(25$ th percentage and 75 th percentage) for non-normally distributed continuous variables, mean \pm SD for normally distributed continuous variables, or frequencies for categorical variables. The significant differences between tertiles were tested using the Kruskal-Wallis tests for non-normally distributed continuous variables, ANOVA for normally distributed continuous variables, and the $\chi^{2}$ tests for categorical variables. ${ }^{2}$ Ranges for tertiles (T) 1 through $3 .{ }^{3}$ Values are given as median (25th percentage and 75 th percentage) of television viewing (in hours/ day). ${ }^{4} \mathrm{GRS}_{\text {weighted }}$ for BMI. ${ }^{5} \mathrm{MVPA}$ energy expenditure: energy expended on MVPAs (MET-h/week) [15, 16]. ${ }^{6} \mathrm{BMI} \geq 25 \mathrm{~kg} / \mathrm{m}^{2}[17]$. ${ }^{7}$ Calculated according to the equations from Liu et al. [18]. ${ }^{8}$ Monthly family income at least $\geq 3,000 \mathrm{CNY}$, which is a moderate level among the general population in Southwest China [12] ${ }^{9}$ At least 12 years of school education.

only found for adults with a genetic predisposition to having a high BMI (Table 4 and online suppl. Tables1-3; see www.karger.com/doi/10.1159/000515380 for all online suppl. material). In the group with a genetic predisposition to having a high BMI, MVPA was negatively associated with $\mathrm{WC}$ and \%BF and positively related to FFMI.

Television watching was positively associated with $\mathrm{BMI}, \mathrm{WC}, \% \mathrm{BF}$, and FMI after adjusting for age, gender, the family's average monthly income, and smoking. The association remained significant, even after including EI and MVPA in the final model (for BMI $0.1 \mathrm{~kg} / \mathrm{m}^{2}, \mathrm{SE}=$ $0.1 \mathrm{~kg} / \mathrm{m}^{2}$; for WC $0.2 \mathrm{~cm}, \mathrm{SE}=0.2 \mathrm{~cm}$; for $\% B F \quad 0.2 \%$, $\mathrm{SE}=0.1 \%$; for FMI $0.1 \mathrm{~kg} / \mathrm{m}^{2}, \mathrm{SE}=0.04 \mathrm{~kg} / \mathrm{m}^{2}$; all $p$ values $<0.04$; Table 5). When the GRS was weighted for obesity, positive associations between the time spent watching television and the BMI, WC, \%BF, and FMI indices were only found among adults with a genetic predisposition to having a high BMI, after adjusting for age, gender, the family's average monthly income, and smoking $(p<0.04$; Table 6 and online suppl. Table 4). Additionally, these positive associations remained significant, even after additionally adjusting for EI and MVPA energy expenditure concurrently. That is, for every $1 \mathrm{~h}$ increment in television watching, BMI, WC, \%BF, and FMI increased by $0.2 \mathrm{~kg} /$ $\mathrm{m}^{2}, 0.9 \mathrm{~cm}, 0.3 \%$, and $0.1 \mathrm{~kg} / \mathrm{m}^{2}$, respectively (all $p$ values $<0.02$; Table 6 ). The time spent on computer/phone use, screen-based SBs, and the total SB time were not associated with body composition when GRS weighted for obesity was considered (online suppl. Tables 5-7).

\section{Discussion}

Our study provided evidence that more MPA may be the protective factor for adiposity, while Chinese adults along with prolonged television watching had higher 
Table 3. Multiple linear regression for the associations between MVPA, MPA, and VPA (MET-h/week) and the body composition indices $(n=3,976)$

\begin{tabular}{|c|c|c|c|c|c|c|}
\hline \multirow[b]{2}{*}{ BMI, $\mathrm{kg} / \mathrm{m}^{2}, \times 10^{-2}$} & \multicolumn{2}{|l|}{ MVPA } & \multicolumn{2}{|l|}{ MPA } & \multicolumn{2}{|l|}{ VPA } \\
\hline & $\beta(\mathrm{SE})$ & $p_{\text {for trend }}$ & $\beta(\mathrm{SE})$ & $p_{\text {for trend }}$ & $\beta(\mathrm{SE})$ & $p_{\text {for trend }}$ \\
\hline Model 1 & $-0.0(0.2)$ & 0.9 & $-0.5(0.2)$ & 0.03 & $0.3(0.2)$ & 0.2 \\
\hline Model 2 & $-0.0(0.2)$ & 0.9 & $-0.5(0.2)$ & 0.02 & $0.3(0.2)$ & 0.2 \\
\hline Model 3 & $-0.0(0.2)$ & 0.8 & $-0.6(0.2)$ & 0.02 & $0.2(0.2)$ & 0.2 \\
\hline \multicolumn{7}{|l|}{$\mathrm{WC}, \mathrm{cm}, \times 10^{-2}$} \\
\hline Model 1 & $-1.1(0.4)$ & 0.01 & $-2.5(0.6)$ & $<0.0001$ & $0.1(0.5)$ & 0.9 \\
\hline Model 2 & $-1.1(0.4)$ & 0.01 & $-2.6(0.6)$ & $<0.0001$ & $0.1(0.5)$ & 0.9 \\
\hline Model 3 & $-1.1(0.4)$ & 0.01 & $-2.5(0.6)$ & $<0.0001$ & $0.1(0.5)$ & 0.8 \\
\hline \multicolumn{7}{|l|}{$\% \mathrm{BF}, \times 10^{-2}$} \\
\hline Model 1 & $-0.2(0.2)$ & 0.1 & $-0.8(0.2)$ & 0.0006 & $0.1(0.2)$ & 0.4 \\
\hline Model 2 & $-0.3(0.2)$ & 0.1 & $-0.9(0.2)$ & 0.0005 & $0.1(0.2)$ & 0.4 \\
\hline Model 3 & $-0.3(0.2)$ & 0.1 & $-0.9(0.2)$ & 0.0004 & $0.1(0.2)$ & 0.4 \\
\hline \multicolumn{7}{|l|}{ FMI, $\mathrm{kg} / \mathrm{m}^{2}, \times 10^{-2}$} \\
\hline Model 1 & $-0.1(0.1)$ & 0.5 & $-0.4(0.1)$ & 0.005 & $0.1(0.1)$ & 0.2 \\
\hline Model 2 & $-0.1(0.1)$ & 0.4 & $-0.4(0.1)$ & 0.005 & $0.1(0.1)$ & 0.2 \\
\hline Model 3 & $-0.1(0.1)$ & 0.4 & $-0.4(0.1)$ & 0.004 & $0.1(0.1)$ & 0.2 \\
\hline \multicolumn{7}{|l|}{ FFMI, $\mathrm{kg} / \mathrm{m}^{2}, \times 10^{-2}$} \\
\hline Model 1 & $0.0(0.1)$ & 0.6 & $-0.2(0.1)$ & 0.1 & $0.1(0.1)$ & 0.1 \\
\hline Model 2 & $0.0(0.1)$ & 0.6 & $-0.2(0.1)$ & 0.1 & $0.1(0.1)$ & 0.1 \\
\hline Model 3 & $0.0(0.1)$ & 0.7 & $-0.2(0.1)$ & 0.1 & $0.1(0.1)$ & 0.1 \\
\hline
\end{tabular}

Values are given as estimate (SE), and linear trends ( $\left.p_{\text {for trend }}\right)$ were obtained with time spent watching television as a continuous variables. Model 1 included adjustment for age at examination (years), gender, the family's monthly income, and smoking; model 2 additionally adjusted for the total EI (kcal/day); model 3 additionally adjusted for SBs (hours/day). WC, waist circumference; VPA, vigorous physical activity; FMI, fat mass index; FFMI, fat-free mass index; \%BF, percentage of body fat; SE, standard error; EI, energy intake; SB, sedentary behavior.

body composition indices, such as BMI, \%BF, WC, and FMI, whereas VPAs were positively related to body composition indices; furthermore, these putative effects could

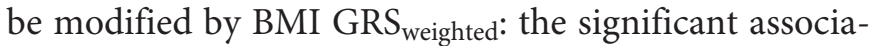
tions between MVPA, time spent on television watching, and body composition indices were only observed among

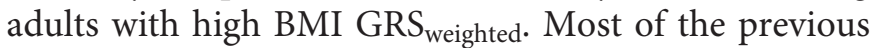
cross-sectional studies that reported the interaction between PA and genetic susceptibility for obesity focused on a single locus, namely, the FTO gene, the so-called obesity gene, which is different from BMI-related genes. In the present study, genetic predisposition was estimated using 9 well-established BMI-associated variants for the Chinese Han population, which reflected the relative overall genetic susceptibility. This approach may be preferable when analyzing the interaction between genetic predisposition and lifestyle factors. The present results are consistent with 2 studies of Chinese adults and suggested that adopting a physically active lifestyle may help to reduce the genetic influence on BMI. It is noteworthy that the negative associations between MVPA and body composition indices were only found among adults with high GRS, which suggests that for people with high genetic risk of obesity, increasing the activities level of MVPA could just in theory effectively slow the increase of obesity-related indicators. While this study is a crosssectional study and the appraisal tool suffered from systematic errors in the PA measurements and the recall errors of the respondents, among other limitations, the effect of MVPA on the body composition indicators was statistically significant, though the average change in obesity indicators was relatively small. More studies are needed to further verify the results of this study.

Remarkably, we found that only MPA was negatively correlated with the body composition indices, whereas the relation of VPA to obesity-related indicators was positive among people with high genetic risk, which is inconsistent with the 2 aforementioned studies of Chinese 
Table 4. Multiple linear regression for the associations of MVPA, MPA, and VPA (MET-h/week) with the body composition indices among adults with a high weighted genetic predisposition score for a high $\mathrm{BMI}(n=1,546)$

\begin{tabular}{|c|c|c|c|c|c|c|}
\hline BMI, $\mathrm{kg} / \mathrm{m}^{2}, \times 10^{-2}$ & $\begin{array}{l}\text { MVPA } \\
\beta(\mathrm{SE})\end{array}$ & $p_{\text {for trend }}$ & $\begin{array}{l}\text { MPA } \\
\beta(\mathrm{SE})\end{array}$ & $p_{\text {for trend }}$ & $\begin{array}{l}\mathrm{VPA} \\
\beta(\mathrm{SE})\end{array}$ & $p_{\text {for trend }}$ \\
\hline Model 1 & $-0.3(0.3)$ & 0.3 & $-1.4(0.4)$ & 0.0002 & $0.7(0.3)$ & 0.03 \\
\hline Model 2 & $-0.3(0.3)$ & 0.3 & $-1.4(0.4)$ & 0.0002 & $0.8(0.3)$ & 0.02 \\
\hline Model 3 & $-0.3(0.3)$ & 0.4 & $-1.4(0.4)$ & 0.0002 & $0.8(0.3)$ & 0.02 \\
\hline \multicolumn{7}{|l|}{$\mathrm{WC}, \mathrm{cm}, \times 10^{-2}$} \\
\hline Model 1 & $-2.4(0.9)$ & 0.006 & $-5.1(1.0)$ & $<0.0001$ & $1.4(0.9)$ & 0.1 \\
\hline Model 2 & $-2.4(0.9)$ & 0.007 & $-5.1(1.0)$ & $<0.0001$ & $1.4(0.9)$ & 0.1 \\
\hline Model 3 & $-2.3(0.9)$ & 0.01 & $-5.0(1.0)$ & $<0.0001$ & $1.6(0.9)$ & 0.08 \\
\hline \multicolumn{7}{|l|}{$\% \mathrm{BF}, \times 10^{-2}$} \\
\hline Model 1 & $-0.7(0.3)$ & 0.03 & $-1.8(0.4)$ & $<0.0001$ & $0.6(0.3)$ & 0.09 \\
\hline Model 2 & $-0.7(0.3)$ & 0.03 & $-1.8(0.4)$ & $<0.0001$ & $0.6(0.3)$ & 0.08 \\
\hline Model 3 & $-0.7(0.3)$ & 0.04 & $-1.8(0.4)$ & $<0.0001$ & $0.6(0.4)$ & 0.07 \\
\hline \multicolumn{7}{|l|}{ FMI, $\mathrm{kg} / \mathrm{m}^{2}, \times 10^{-2}$} \\
\hline Model 1 & $-0.3(0.2)$ & 0.1 & $-0.8(0.2)$ & $<0.0001$ & $0.4(0.2)$ & 0.053 \\
\hline Model 2 & $-0.3(0.2)$ & 0.1 & $-0.8(0.2)$ & $<0.0001$ & $0.4(02)$ & 0.04 \\
\hline Model 3 & $-0.3(0.2)$ & 0.2 & $-0.8(0.2)$ & $<0.0001$ & $0.4(0.2)$ & 0.03 \\
\hline \multicolumn{7}{|l|}{ FFMI, $\mathrm{kg} / \mathrm{m}^{2}, \times 10^{-2}$} \\
\hline Model 1 & $-0.1(0.2)$ & 0.7 & $-0.1(0.2)$ & 0.3 & $0.4(0.2)$ & 0.02 \\
\hline Model 2 & $-0.1(0.2)$ & 0.7 & $-0.1(0.2)$ & 0.3 & $0.4(0.2)$ & 0.01 \\
\hline Model 3 & $-0.1(0.2)$ & 0.7 & $-0.0(0.2)$ & 0.3 & $0.4(0.2)$ & 0.01 \\
\hline
\end{tabular}

Values are given as estimate (SE), and linear trends $\left(p_{\text {for trend }}\right)$ were obtained with time spent watching television as a continuous variables; model 1 included adjustment for age at examination (years), gender, the family's monthly income, and smoking; model 2 additionally adjusted for the total EI (kcal/day); model 3 additionally adjusted for SBs (hours/day). WC, waist circumference; VPA, vigorous physical activity; FMI, fat mass index; FFMI, fat-free mass index; \%BF, percentage of body fat; SE, standard error; EI, energy intake; SB, sedentary behavior.

adults $[8,9]$. One possibility is that MPA may generate low levels of reactive oxygen species that induce adaptive increases in endogenous antioxidant defenses, while VPA may generate excessive reactive oxygen species that counteract these defenses [30], which might be a possibility and need to be validated. Additionally, people with high GRS were more likely to be obese, while people with relatively high body weight may tend to undergo the highintensity physical exercise to lose weight, which masks the fact that they are less physically active. Furthermore, differences in the population, the GRS based on different genes, different PA questionnaire items, different study times, and differences in controlled confounding factors in the analysis models could have led to differences in the study results. Moreover, as this study is cross-sectional, the causal relationship between PA and obesity cannot be determined, and more studies are needed to verify the relevant mechanism(s).
There is a coherent body of evidence based on observational studies from the past 15 years that documents the deleterious health effects of SBs. Although many publications from Western countries have suggested that the most common SBs, namely, television watching, may be a risk factor for adulthood obesity [5, 11, 12, 31-33], this remains to be determined in China. To date, only 2 crosssectional studies on adults -1 in Hong Kong and 1 in rural China - have examined the relationship between television watching and body composition, where these studies have suggested positive association of television watching with BMI and WC. However, it is noteworthy that BMI and WC are only surrogate measure of body fatness [34]. Obesity is defined as an excess accumulation of body fat, and it is the amount of this excess fat that correlates with ill health. Thus, unlike aforementioned 2 studies, the present study not only focused on BMI and WC but also considered relationship between the body fat measures, namely \%BF, FMI, and FFMI. The present 
Table 5. Multiple linear regression for the associations between time spent on watching television (hours/day) and the body composition indices $(n=3,976)$

\begin{tabular}{rll}
\hline BMI, kg/m ${ }^{2}$ & $\beta(\mathrm{SE})$ & $p_{\text {for trend }}$ \\
\hline Model 1 & $0.1(0.1)$ & 0.04 \\
Model 2 & $0.1(0.1)$ & 0.058 \\
Model 3 & $0.1(0.1)$ & 0.03 \\
WC, cm & & \\
Model 1 & $0.2(0.1)$ & 0.02 \\
Model 2 & $0.2(0.1)$ & 0.055 \\
Model 3 & $0.2(0.2)$ & 0.03 \\
\% BF & & \\
Model 1 & $0.5(0.1)$ & 0.04 \\
Model 2 & $0.5(0.1)$ & 0.04 \\
Model 3 & $0.2(0.1)$ & 0.04 \\
FMI, kg/m ${ }^{2}$ & & \\
Model 1 & $0.1(0.0)$ & 0.04 \\
Model 2 & $0.1(0.0)$ & 0.09 \\
Model 3 & $0.1(0.0)$ & 0.04 \\
FFMI, kg/m ${ }^{2}$ & & \\
Model 1 & $0.0(0.0)$ & 0.4 \\
Model 2 & $0.0(0.0)$ & 0.09 \\
Model 3 & $0.1(0.0)$ & 0.2
\end{tabular}

Values are given as estimate (SE) and linear trends ( $p_{\text {for trend }}$ ) were obtained with time spent watching television as a continuous variable; model 1 included adjustment for age at examination (years), gender, the family's average monthly income, and smoking; model 2 additionally adjusted for total EI (kcal/day); model 3 additionally adjusted for MVPA energy expenditure (MET-h/ week). WC, waist circumference; FMI, fat mass index; FFMI, fatfree mass index; \%BF, percentage of body fat; SE, standard error; EI, energy intake.

study showed that the total sedentary time, screen-based sedentary time, and time on a computer/phone were not related to the body composition indices. In accordance with existing studies [35], more time spent watching television was related to a high BMI and WC among Chinese adults. Concurrently, we observed that television watching was positively associated with \%BF and FMI but not with FFMI. As we mentioned in another article, which investigated the relationship between SBs and telomere length (also closely related to lifestyle [19]), possible reasons for the above-mentioned results are as follows: (1) the concurrent intake of unhealthy foods with television watching and (2) sleeping late due to watching television late at night, which potentially affects sleep quality [36] - another important factor contributing to obesity [37]. Another reason may be due to the nature of this study, that is, cross-sectional, and the phenomenon of obese people controlling their weight by reducing the amount
Table 6. Multiple linear regression for the associations between time spent watching television (hours/day) and the body composition indices among adults with a high weighted genetic predisposition score for a high BMI $(n=1,307)$

\begin{tabular}{rll}
\hline BMI, kg/m ${ }^{2}$ & \multicolumn{2}{l}{ High GRS for a high BMI } \\
\cline { 2 - 3 } & $\beta(\mathrm{SE})$ & $p_{\text {for trend }}$ \\
\hline Model 1 & $0.2(0.1)$ & 0.03 \\
Model 2 & $0.2(0.1)$ & 0.02 \\
Model 3 & $0.2(0.1)$ & 0.02 \\
WC, cm & & \\
Model 1 & $0.7(0.2)$ & 0.03 \\
Model 2 & $0.9(0.2)$ & 0.0004 \\
Model 3 & $0.9(0.2)$ & 0.0004 \\
\% BF & & \\
Model 1 & $0.2(0.1)$ & 0.02 \\
Model 2 & $0.3(0.1)$ & 0.003 \\
Model 3 & $0.3(0.1)$ & 0.003 \\
FMI, kg/m 2 & \\
Model 1 & $0.1(0.0)$ & 0.04 \\
Model 2 & $0.1(0.0)$ & 0.005 \\
Model 3 & $0.1(0.0)$ & 0.006 \\
FFMI, kg/m ${ }^{2}$ & & \\
Model 1 & $0.0(0.0)$ & 0.3 \\
Model 2 & $0.1(0.0)$ & 0.07 \\
Model 3 & $0.1(0.0)$ & 0.08 \\
\hline
\end{tabular}

Values are given as estimate (SE), and linear trends $\left(p_{\text {for trend }}\right)$ were obtained with time spent watching television as a continuous variable; model 1 included adjustment for age at examination (years), gender, the family's average monthly income, and smoking; model 2 additionally adjusted for total EI (kcal/day); model 3 additionally adjusted for MVPA energy expenditure (MET-h/ week). GRS, genetic risk score; WC, waist circumference; FMI, fat mass index; FFMI, fat-free mass index; \%BF, percentage of body fat; SE, standard error; EI, energy intake.

of time they spend in a static lifestyle appearing at the selected time node.

To date, only 2 studies - 1 from the USA [11] and 1 from Finland [12] - have investigated the interacting effect of television watching and genetic predisposition on body composition indices. The US study [11] showed that prolonged television viewing by women aged $30-55$ years and men aged $40-75$ years may increase the tendency toward a higher BMI. The study focused on Finnish adults aged 30-45 years [12] showed a positive correlation between television viewing and BMI and WC, even after adjusting for the GRS for obesity [7]. In the present analysis, we observed an interaction effect of time spent watching television and genetic predisposition on the body composition indices, not only BMI and WC but also 
\%BF and FMI. That is to say, in this study, the relationship between television watching and these body composition indices differed among Chinese adults with different genetic predispositions. Positive significant associations were only observed among those individuals with a high $\mathrm{GRS}_{\text {weighted }}$ for a high BMI, although it was not significant among participants with a low or moderate GRSweighted.

The time spent watching television is an indicator that scholars pay close attention to when studying sedentary lifestyles. However, television viewing time is affected by many confounding factors, where we adjusted for many of them, such as EI and PAs. It is worth noting that the obesity-inducing effects of television watching time may also be related to poor dietary habits, which remain difficult to accurately and comprehensively measure in large epidemiological studies. In this study, due to the limitations of the sample population and to ensure fitting effects of the statistical models, the influence of dietary habits on the relationship between television watching time and obesity was not adjusted, which may be one of the important factors that affected the results of this study. Additionally, a study found that baseline levels of obesity were predictive of the sedentary lifestyle time in the population at follow-up, the GRS of people with a higher BMI were closely related to sitting time, and obese people may be too heavy to exercise and sit for longer periods [38]. The present results hinted that the relationship between television watching time and body composition was accentuated in adults with a high genetic predisposition to obesity, which might be an important group for which targeted SB interventions should be developed, implemented, and evaluated.

Although this study was cross-sectional, the present findings may have important public health implications because we showed that SBs (as indicated by prolonged television watching) may accentuate the genetic effects on the body composition indices. In contrast, more MVPA may attenuate the genetic predisposition to increased adiposity. These results evidence that the deleterious effects of genetic factors could be modified (attenuated or accentuated) by lifestyle factors and challenge the common notion that a genetic predisposition to obesity cannot be overcome. The present data strongly suggest that a more active lifestyle, with both increased exercise and reduced SBs in leisure time, especially television watching, is critical for reducing obesity risk, especially among those with a high genetic risk.

Several of the considerable strengths of our study should be mentioned, including the detailed assessment of SBs, PAs, dietary intake, and lifestyle variables, which were collected by trained investigators in face-to-face interviews. Particularly, unlike other studies that used a family history questionnaire to characterize the genetic backgrounds of participants, we characterized our participants using a GRS that combined the genetic information of variants associated with BMI among Chinese adults. A further advantage is in the use of comprehensive markers to characterize body composition. Several drawbacks need to be mentioned: (1) the cross-sectional design of the study means that the causality of the effect of SBs on body composition measures could not be determined; (2) the small sample size of this analysis for Chinese adults, which might decrease the statistical power of the findings; and (3) the presence of unmeasured or residual confounding variables remains a possibility.

In conclusion, our study provided evidence that MPA may be a protective factor against obesity, while prolonged television watching by Chinese adults was associated with higher body composition indices, namely BMI, $\% \mathrm{BF}, \mathrm{WC}$, and FMI. Moreover, VPA was positively related to the body composition indices. These putative ef-

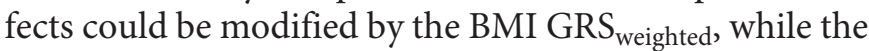
significant associations between MVPA, the time spent on television watching, and the body composition indices were only observed among adults with a high BMI GRSweighted

\section{Acknowledgements}

We gratefully acknowledge all participants and participant advisers. We thank the staff of the Department of Nutrition, Food Safety and Toxicology for organizing this study and the laboratorian of No. 4 West China Teaching Hospital for providing technical support.

\section{Statement of Ethics}

This study was conducted according to the guidelines laid down in the Declaration of Helsinki, and all procedures involving human subjects were approved by the Ethics Committee of West China School of Public Health (No. 4 West China Teaching Hospital) of Sichuan University (approved date: March 3, 2014). Written informed consent was obtained from all the participants before they took part in the measurement procedures.

\section{Conflict of Interest Statement}

The authors have no conflicts of interest to declare. 


\section{Funding Sources}

This work was supported by a research grant from the National Nature Science Foundation of China (Grant No. 81472976). The funders had no additional role in the design, analysis, or writing of this article.

\section{Author Contributions}

G.C. conceptualized the project and designed the study. H.X. conducted the analyses. H.X. and X.Z. wrote the manuscript. M.C. provided dietary data and critical input on the data analyses. H.X., M.C., and J.L. extracted the genomic DNA and provided genetic data. Y.G., X.L., and L.Q. coordinated the study centers. All authors critically reviewed the manuscript for important intellectual content.

\section{References}

1 World Health Organization. Global Health Observatory data. [cited 2018 Dec 5]. Available from: http://www.who.int/gho/ncd/ risk_factors/overweight/en/.

2 Boutin P, Froguel P. Genetics of human obesity. Best Pract Res Clin Endocrinol Metab. 2001;15(3):391-404.

3 Chin SH, Kahathuduwa CN, Binks M. Physical activities and obesity: what we know and what we need to know. Obes Rev. 2016; 17(12):1226-44.

4 Tucker LA, Tucker JM. Television viewing and obesity in 300 women: evaluation of the pathways of energy intake and physical activities. Obesity. 2011;19(10):1950-6.

5 Bann D, Hire D, Manini T, Cooper R, Botoseneanu A, McDermott MM, et al. Light intensity physical activities and sedentary behaviours in relation to body mass index and grip strength in older adults: cross-sectional findings from the Lifestyle Interventions and Independence for Elders (LIFE) study. PLoS One. 2015;10(2):e0116058.

6 Myers A, Gibbons C, Finlayson G, Blundell J. Associations among sedentary and active behaviours, body fat and appetite dysregulation: investigating the myth of physical inactivities and obesity. Br J Sports Med. 2017;51(21): $1540-4$.

7 Eriksen D, Rosthøj S, Burr H, Holtermann A. Sedentary work: associations between fiveyear changes in occupational sitting time and body mass index. Prev Med. 2015;73:1-5.

8 Wang B, Gao W, Lv J, Yu C, Wang S, Pang Z, et al. Physical activities attenuates genetic effects on BMI: results from a study of Chinese adult twins. Obesity. 2016;24(3):750-6.

9 Zhu J, Loos RJ, Lu L, Zong G, Gan W, Ye X, et al. Associations of genetic risk score with obesity and related traits and the modifying effect of physical activity in a Chinese Han population. PLoS One. 2014;9(3):e91442.

10 Bondia-Pons I, Ryan L, Martinez JA. Oxidative stress and inflammation interactions in human obesity. J Physiol Biochem. 2012; 68(4):701-11.

11 Qi Q, Li Y, Chomistek AK, Kang JH, Curhan GC, Pasquale LR, et al. Television watching, leisure time physical activity, and the genetic predisposition in relation to body mass index in women and men. Circulation. 2012; 126(15):1821-7.
12 Heinonen I, Helajärvi H, Pahkala K, Heinonen OJ, Hirvensalo M, Pälve K, et al. Sedentary behaviours and obesity in adults: the cardiovascular risk in young Finns Study. BMJ Open. 2013;3(6):e002901.

13 Fan M, Lyu J, Guo Y, Bian Z, Yu C, Du H, et al. Regional differences on patterns of physical activities and leisure sedentary time: findings from the China Kadoorie Biobank study, including a million people from 10 regions (Chinese). Zhonghua Liu Xing Bing Xue Za Zhi. 2015;36(8):779-85.

14 Cheung CY, Tso AW, Cheung BM, Xu A, Ong $\mathrm{KL}$, Fong $\mathrm{CH}$, et al. Obesity susceptibility genetic variants identified from recent genomewide association studies: implications in a Chinese population. J Clin Endocrinol Metab. 2010;95(3):1395-403.

15 Liu Y, Liu Z, Song Y, Zhou D, Zhang D, Zhao $\mathrm{T}$, et al. Meta-analysis added power to identify variants in FTO associated with type 2 diabetes and obesity in the Asian population. Obesity. 2010;18(8):1619-24.

16 Zhang X, Chen M, Duan R, Xue H, Luo J, Lv $\mathrm{X}$, et al. The Nutrition and Health in Southwest China (NHSC) study: design, implementation, and major findings. Eur J Clin Nutr. 2020. Online ahead of print.

17 Sichuan Statistical Yearbook-2013 Editorial Board and Staff. p. 5.

18 Yu D, Zhang X, Xiang YB, Yang G, Li H, Gao $\mathrm{YT}$, et al. Adherence to dietary guidelines and mortality: a report from prospective cohort studies of 134,000 Chinese adults in urban Shanghai. Am J Clin Nutr. 2014;100(2):693700 .

19 Xue HM, Liu QQ, Tian G, Quan LM, Zhao Y, Cheng G. Television watching and telomere length among adults in Southwest China. Am J Public Health. 2017 Sep;107(9):1425-32.

20 Ainsworth BE, Haskell WL, Herrmann SD, Meckes N, Bassett DR Jr, Tudor-Locke C, et al. 2011 compendium of physical activities: a second update of codes and MET values. Med Sci Sports Exerc. 2011;43(8):1575-81.

21 Ainsworth BE, Haskell WL, Leon AS, Jacobs DR Jr, Montoye HJ, Sallis JF, et al. Compendium of physical activities: classification of energy costs of human physical activities. Med Sci Sports Exerc. 1993;25(1):71-80.
22 Jensen MD, Ryan DH, Apovian CM, Ard JD, Comuzzie AG, Donato KA, et al. 2013 AHA/ ACC/TOS guideline for the management of overweight and obesity in adults: a report of the American College of Cardiology/American Heart Association Task Force on Practice Guidelines and The Obesity Society. J Am Coll Cardiol. 2014;63(25 Suppl 2):2985-3023.

23 Liu X, Sun Q, Sun L, Zong G, Lu L, Liu G, et al. The development and validation of new equations for estimating body fat percentage among Chinese men and women. Br J Nutr. 2015;113(9):1365-72.

24 VanItallie TB, Yang MU, Heymsfield SB, Funk RC, Boileau RA. Height-normalized indices of the body's fat-free mass and fat mass: potentially useful indicators of nutritional status. Am J Clin Nutr. 1990;52(6):953-9.

25 Hosking L, Lumsden S, Lewis K, Yeo A, McCarthy L, Bansal A, et al. Detection of genotyping errors by Hardy-Weinberg equilibrium testing. Eur J Hum Genet. 2004;12(5): 395-9.

26 Cornelis MC, Qi L, Zhang C, Kraft P, Manson $\mathrm{J}$, Cai T, et al. Joint effects of common genetic variants on the risk for type 2 diabetes in U.S. men and women of European ancestry. Ann Intern Med. 2009;150(8):541-50.

27 De Jager PL, Chibnik LB, Cui J, Reischl J, Lehr $\mathrm{S}$, Simon KC, et al. Integration of genetic risk factors into a clinical algorithm for multiple sclerosis susceptibility: a weighted genetic risk score. Lancet Neurol. 2009;8(12):1111-9.

28 Talmud PJ, Hingorani AD, Cooper JA, Marmot MG, Brunner EJ, Kumari M, et al. Utility of genetic and non-genetic risk factors in prediction of type 2 diabetes: Whitehall II prospective cohort study. BMJ. 2010;340:b4838.

29 Yang YX, Wang GY, Pang XC. China food composition. 2nd ed. Beijing: Peking University Medical Press; 2009.

30 Sallam N, Laher I. Exercise modulates oxidative stress and inflammation in aging and cardiovascular diseases. Oxid Med Cell Longev. 2016;2016:7239639.

31 Maher CA, Mire E, Harrington DM, Staiano AE, Katzmarzyk PT. The independent and combined associations of physical activities and sedentary behaviours with obesity in adults: NHANES 2003-06. Obesity. 2013; 21(12):E730-7. 
32 Stamatakis E, Hamer M, Tilling K, Lawlor DA. Sedentary time in relation to cardio-metabolic risk factors: differential associations for self-report vs accelerometry in working age adults. Int J Epidemiol. 2012;41(5):1328-37.

33 Pinto Pereira SM, Power C. Sedentary behaviours in mid-adulthood and subsequent body mass index. PLoS One. 2013;8(6):e65791.
34 Prentice AM, Jebb SA. Beyond body mass index. Obes Rev. 2001;2(3):141-7.

35 Richard MP, Emmanuel S, Annie RB, Eric JB, Melvyn MH. Sitting behavior and obesity: evidence from the Whitehall II study. Am J Prev Med. 2013;44(2):132-8.

36 Hart CN, Hawley N, Davey A, Carskadon M, Raynor H, Jelalian E, et al. Effect of experimental change in children's sleep duration on television viewing and physical activity. Pediatr Obes. 2017;12(6):462-7.
37 Rahe C, Czira ME, Teismann H, Berger K. Associations between poor sleep quality and different measures of obesity. Sleep Med. 2015; 16(10):1225-8.

38 Ekelund U, Brage S, Besson H, Sharp S, Wareham NJ. Time spent being sedentary and weight gain in healthy adults: reverse or bidirectional causality? Am J Clin Nutr. 2008; $88(3): 612-7$ 